\title{
Cooperative Content Downloading Framework over Cellular Network
}

\author{
Niranjan Armal \\ Department of Computer Engineering \\ Dr, D.Y.Patil SOET, \\ Lohegaon, Pune
}

\author{
Aarti Mohanpukar \\ HOD, Department of Computer Engineering \\ Dr, D.Y.Patil SOET, \\ Lohegaon, Pune
}

\begin{abstract}
The development in wireless communication technology and mobile computing has made it possible to use smart phones, laptops, and tabs massively for downloading purpose. There are a number of applications which provide a single user downloading scheme but there is no provision for cooperative downloading i.e. multiple user can download the same file provided each group member will download a separate segment of file. The software likes Torrent, YouTube facilitates only a single end user to download a file. However because of limited bandwidth access the data download volume by an individual is quite less and take much more time to download a high resolution video. Nowadays, there are unlimited $2 \mathrm{G} / 3 \mathrm{G}$ plans provided by internet service provider the most of data plans are waste due to bandwidth limitations. The cooperative content downloading framework will allow the requested members in the group to download some portion of the file separately. This will provide the ability to download the file cooperatively in less time and cost. The proposed system will also show the actual process how the bandwidth can be shared among the friends and group member. The system will provide the efficient use of bandwidth in different environment.
\end{abstract}

\section{General Term}

Cooperative Downloading

\section{Keywords}

Segmentation, Group formation, Adhoc network, Sequencing.

\section{INTRODUCTION}

Nowadays the internet service provider gives the unlimited data pack, maybe it is for wired or wireless devices like mobile or laptops and desktop. Most data planes are wasted due to the limited bandwidth offered by an internet service provider. An individual can't download a huge file because of his limited bandwidth. So in order to make efficient use of available resource the proposed system states a method called cooperative downloading. In this the multiple members can take part in downloading single file. Here each member is responsible for downloading some portion of desired file independently, with using his own bandwidth and hardware device.

The proposed system can be used in VANET, LAN or mobile adhoc network for any kind of downloading. We focus on how to form a chain topology before coverage to keep it stable. Wireless cooperative network architecture, where a group of users use dedicated short range communication to save the costs of a cellular download. To maximize the efficiency of the communication system, an optimization of parameters such as download time, monetary cost can be implemented. Following these approach different portions of data shall be assigned for download to the involved users, which will then cooperatively exchange the contents on the adhoc network which will have no concern with internet.

The method of task assignment to the user terminals has a direct influence on the payoff of the single users, raising fairness issues in real implementation scenarios. While one end user can take out best of available bandwidth and data pack plan provided by internet service provider.

The flow of cooperative content downloading framework is in the following stages.

1. Scanning for members.

2. Requesting the members in group.

3. File segmentation and sequencing.

4. Allocating task.

5. Data collection by using adhoc network.

\section{RELATED WORK}

In this section we survey research issue in the cooperative content downloading framework [1]. Most of researches have state cooperative video streaming technique, which facilitate watching a video cooperatively [2]. But our proposed system will explain the segmentation and sequencing technique for desire approached work. Most of the work of downloading is done by considering single user but we will take multiple group members to complete the task [3]. Since the bandwidth of the $3 \mathrm{G} / 3.5 \mathrm{G}$ network over moving vehicular networks is unstable and insufficient, the video quality of the requested video stream may not be good enough. Even using $4 \mathrm{G}$ network, the bandwidth still may not be enough for the following concerns [4].

First, other applications may utilize the 4G network simultaneously. Second, the moving behavior of one vehicle e.g. moving with high speed or around the coverage boundary of one base station makes the decaying of $4 \mathrm{G}$ bandwidth. In order to increase the video quality during the travelling path, one vehicle would ask other vehicles belonging to the same fleet to download video data using their redundant $3 \mathrm{G} / 3.5 \mathrm{G}$ bandwidth.

\subsection{Existing System}

The existing software for downloading facilitates only a single user for downloading. Means a single user can download a single file, we can't cooperatively download a single file or can't share our bandwidth for downloading a file or video. The existing technology provides the multiple storage facilities but doesn't support multiple user downloading. 


\section{SYSTEM MODEL}

The entire system work is describe stage wise, as shown in figure 1 each step is perform respectively.

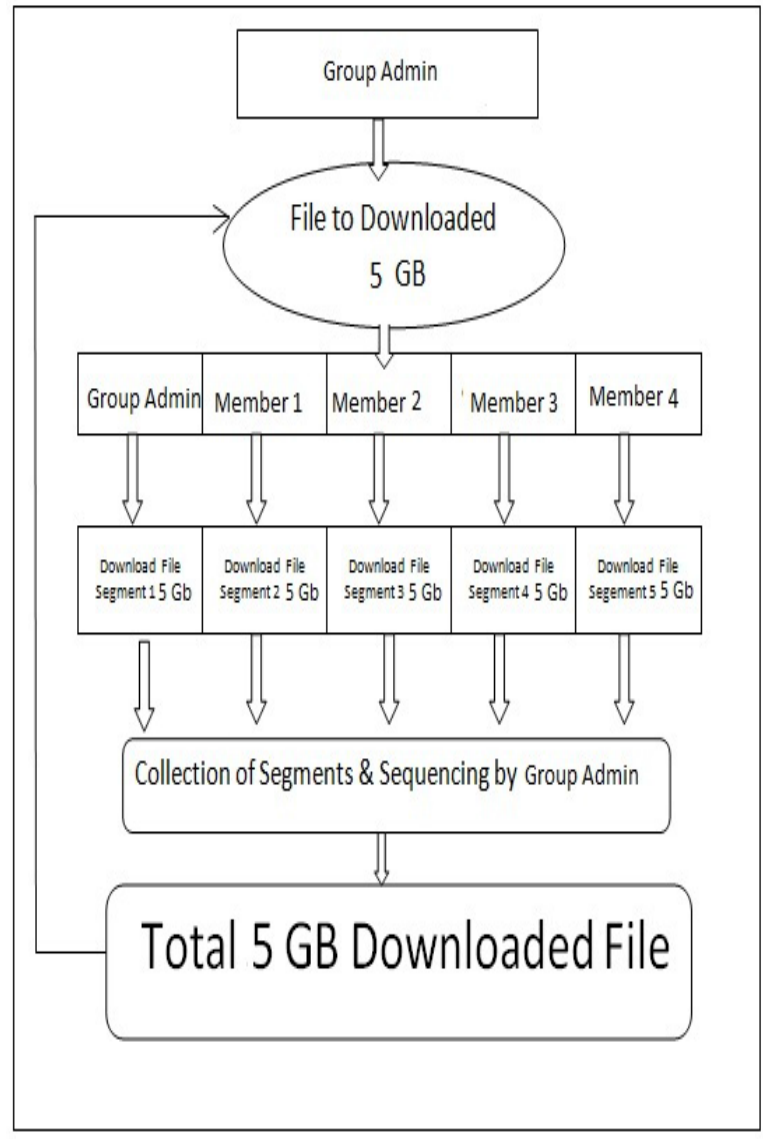

Fig.1 System Architecture

\subsection{Scanning for Members}

The group admin or the user who wants to download a file will scan the members within his proximity who can help to the file. Once he find them through Wi-Fi or Bluetooth, then he will send request to be member of the group to download certain file. As per the interest the member will accept or deny the request for helping the admin. If request is being accepted by him he will be the group member and share his bandwidth to download some portion of file.

\subsection{Requesting the Members}

As per the interest the member will accept or deny the request for helping the admin. If request is being accepted by him he will be the group member and share his bandwidth to download some portion of file. This specifically selects those members which are very closer to Group Admin and has good bandwidth. After getting the acknowledgement from the member the admin will decide how much segments of file should be done.

\subsection{File segmentation and sequencing}

In order to download any file it is break into number of segments and file is rank in particular order, so after receiving it can be arranged properly. Once the acknowledgement has been received the Admin will do the segmentation and allocate the task for downloading. The respective cluster member will download the allocated segment with his own available bandwidth. Here given file as shown in figure 2 first break into five segments and further sequencing is done in numerical format like $1 / 5,2 / 5,3 / 5,4 / 55 / 5$.

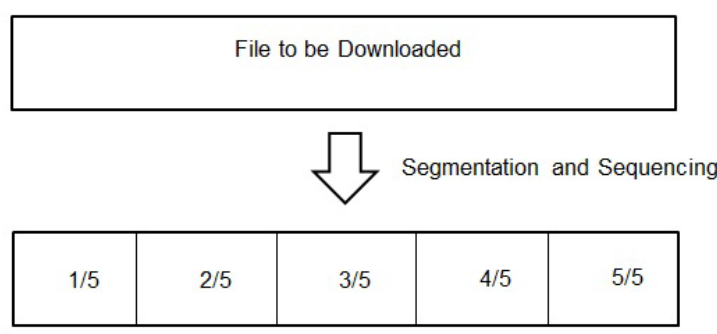

Fig. 2 Segmentation and Sequencing

\subsection{Allocating Task}

Once the file is broke into number of segments, according to the group member availability and the segments which are done the task of downloading is allocated to each member of group. Here each member is responsible for downloading the segment which has been allocated.

\subsection{Data Collection by using Adhoc network}

After downloading the group admin will forward the downloaded data to the Admin through the adhoc network without using the cellular network. Here adhoc network can be of Bluetooth or Wi-Fi depending upon the service available to devices. Then Admin will do the collection of segment form each member and put it in sequence and the file will be ready to use or if the file is video format it is ready to watch.

\section{IMPEMENTATION AND RESULTS}

The below figure is home page of actual project, the left side of GUI is file selection which user want to download. When the user of actual proposed system enters as admin or the user who wants to download file the below tabs are visible. As shown the admin will scan for in the proximity, suppose the four members accept the request for sharing their bandwidth and help for downloading file.

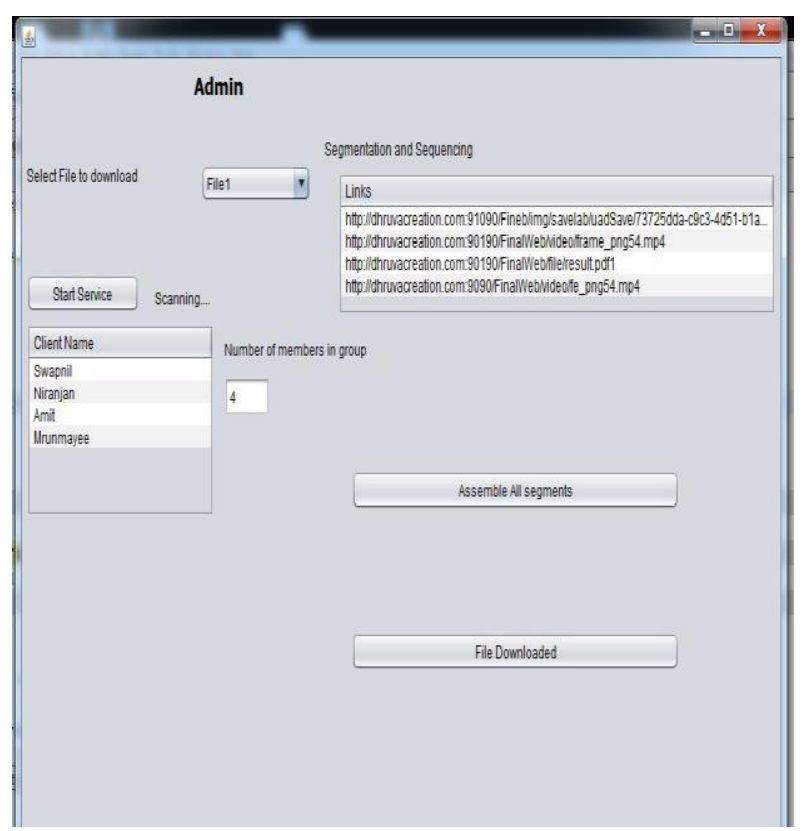

Fig. 3 Admin interface 


\subsection{Admin interface}

The admin makes four segments as four members accept the request to be member of group. The links of this entire segment is seen in admin interface, then as per the sequence of links and member available the link of each segment is forward to the each member respectively. The separate link of the respective segment is received to group member and from the same link he will download the actual segment from the file server. The downloaded file by the member will send to admin by each member. These segments are collected and assemble to make one file which admin wants to download.

\subsection{Members interface}

When an end user don't want to download a file but want help his friend for downloading a file. The end user will enter as member and following interface is available for use. The member only has the authority to accept or deny the request of membership and to download a file from the link which is received from group admin

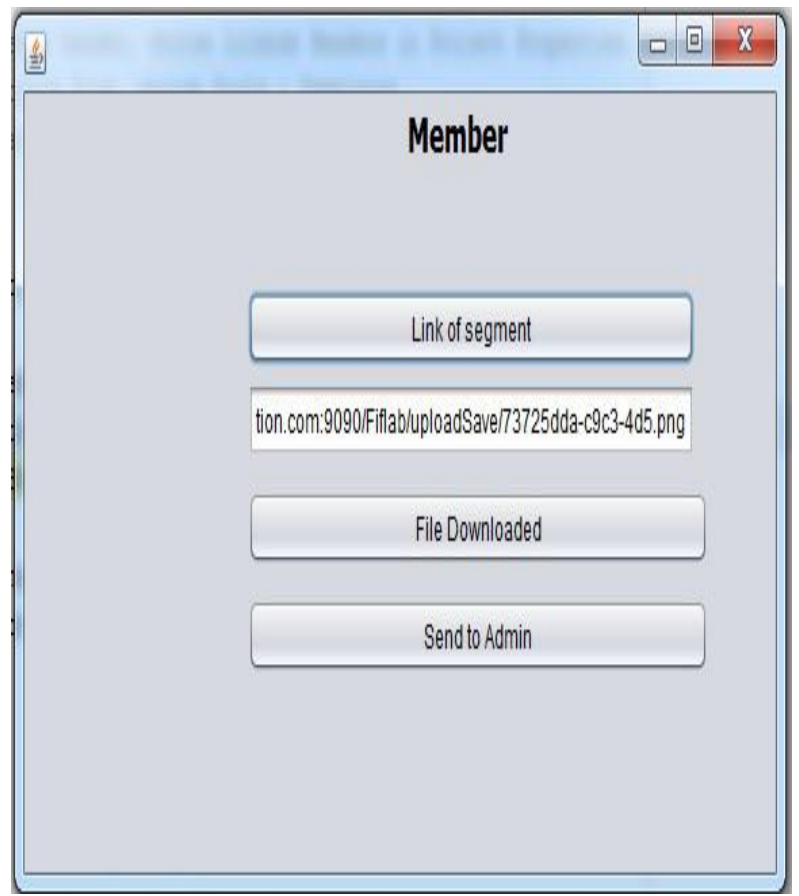

Fig. 4 Member interface

\subsection{Acceptance and denial of request}

The below figure show how an popup message will arrive and at member side, there itself an member can accept or deny the request, same like how Bluetooth provides the function of file sending.

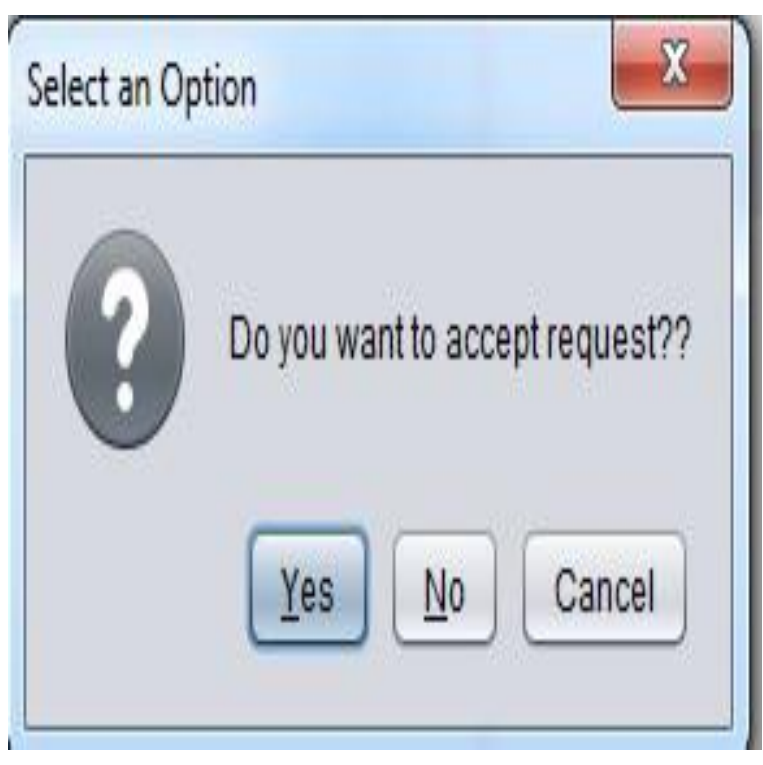

Fig. 5 Request message

If the member clicks on YES then he is be member of group for downloading a file.

\subsection{Segmentation of file}

The file on server which is segmented as per the member in the group is based on the following formula.

$$
\text { Number of Segments }=\frac{\text { FileSize }}{\text { Numberof Member } s}
$$

Here according to the file size, the number segments and the size of each segment an individual user have to download is calculated by using above formula. Larger the size of the file an admin want to download larger the workload will be distributed among the group member.

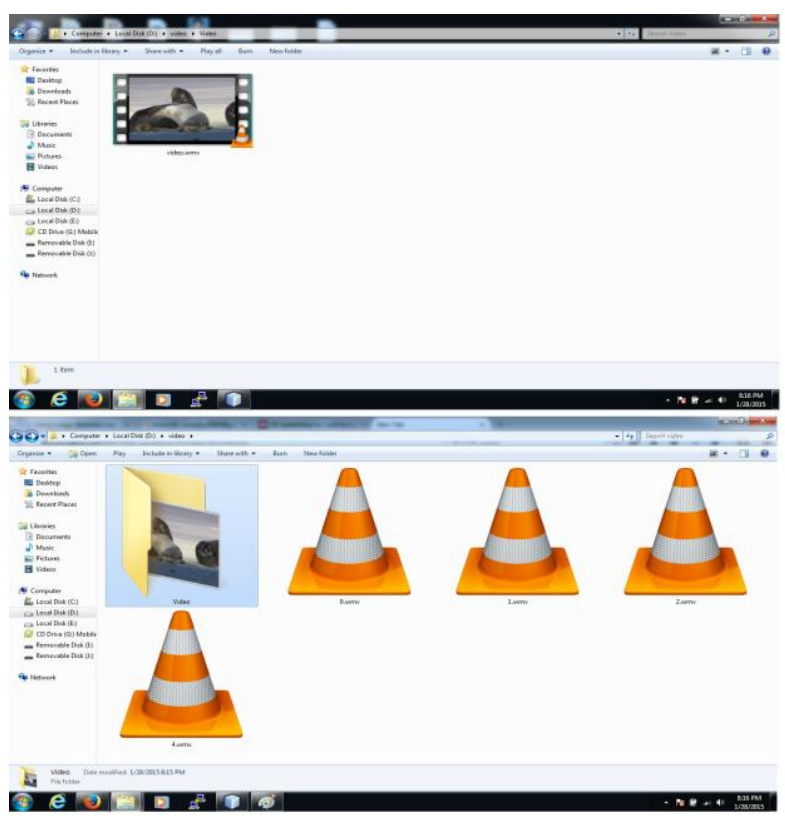

Fig. 6 segmentation of video file 
In above figure the blocks show the actual segmentation of file. One file is segmented into four parts as per the requirement on the server itself.

\section{EXPECTED RESULT ANALYSIS}

Our experiments were conducted by considering various group members in downloading a file.

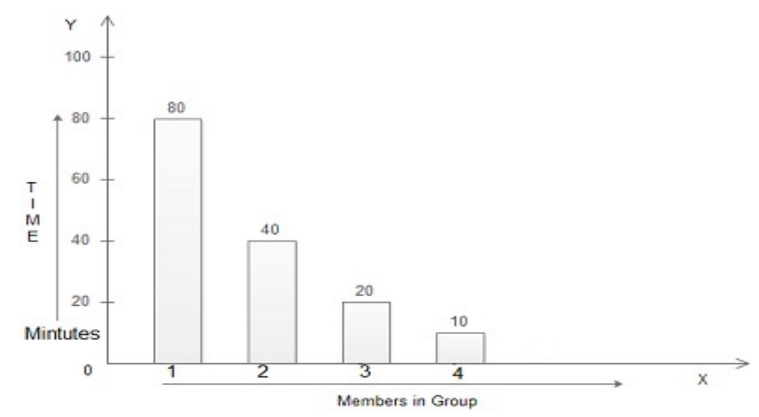

If one member require 80 minutes download a file the same file can be downloading within 10 minutes by group of four friends. The graph shows that as number of member increase time required to download a certain file decrease.

\section{CONCLUSION}

This paper proposed a hybrid cooperative video downloading over the hybrid networks, which is composed of cellular network and wired network. The proposed cooperative video downloading has focused on the issues belonging to the application layer. The technique can be used by many video hosting website and end user which are widely using the cellular bandwidth for downloading purpose. The system had overcome the bandwidth issues for downloading large file in considering multiple obstacles. Same approach can be used for the existing LAN network considering the server as a group Admin and all the remaining desktops as group members. In further research will focus on the issues of network layer for cooperative downloading of file.

\section{ACKNOWLEDGMENT}

We would like to thank to researchers for making their resources available and teachers for guidance, support, suggestions and encouragement.

\section{REFERENCES}

[1] Haibo Zhou, Student Member, IEEE, Bo Liu, Member, IEEE, Tom H. Luan, Member, ChainCluster:
Engineering a Cooperative Content Distribution Framework for Highway Vehicular Communications, IEEE transactions on intelligent transportation systems, 2014.J. Clerk Maxwell, A Treatise on Electricity and Magnetism, 3rd ed., vol. 2. Oxford: Clarendon, 1892, pp.68-73.

[2] Chao-Hsien Lee, Chung-Ming Huang, Senior Member, IEEE, Chia- Ching Yang, and Hsiao-Yu Lin,, The K-hop Cooperative Video Streaming Protocol Using H.264/SVC Over the Hybrid Vehicular Networks, IEEE TRANSACTIONS ON MOBILE COMPUTING, VOL. 13, NO. 6, JUNE 2014.

[3] J. Luo and D. Guo, Neighbor discovery in wireless adhoc networks based on group testing, in Proc. 46th Annu. Allerton Conf. Communication, Control, Computing, Urbana Champaign, IL, USA Sep. 2008, pp. 791797.

[4] R. Khalili, D. L. Goeckel, D. Towsley, and A. Swami, Neighbor discovery with reception status feedback to transmitters, in Proc. $29^{\text {th }}$ IEEE Conf. INFOCOM, San Diego, CA, USA, Mar. 2010,pp. 23752383

[5] C.-M. Huang, C.-C. Yang, and H.-Y. Lin, A K-hop bandwidth aggregation scheme for member-based cooperative transmission over vehicular networks, in Proc. 17th IEEE ICPADS, Tainan, Taiwan, 2011, pp. 436443.

[6] A. Nandan, S. Das, G. Pau, M. Gerla, and M. Y. Sanadidi, Cooperative downloading in vehicular ad-hoc wireless networks, in Proc. 2nd Annu. Conf. WONS, Washington, DC, USA, 2005 pp. 3241.

[7] M. F. Tsai, N. Chilamkurti, J. H. Park, and C. K. Shieh, Multi-path transmission control scheme combining bandwidth aggregation and packet scheduling for realtime streaming in multi-path environment, Instit. Eng. Technol. Commun., vol. 4, no. 8, pp. 937945, 2010.

[8] M. Y. Hsieh, Y. M. Huang, and T. C. Chiang, Transmission of layered video streaming via multi-path on ad-hoc networks.

[9] D. Fan, V. Le, Z. Feng, Z. Hu, and X. Wang, Adaptive joint session scheduling for multimedia services in heterogeneous.

[10] M. Li, Z. Yang, and W. Lou, Codeon: Cooperative popular content distribution for vehicular networks using symbol level network coding, IEEE J. Sel. Areas Commun, vol. 29, no. 1, pp. 223235, Jan. 2011. 